\title{
Experimental Determination of Particle Size Distributions in Colloidal Systems by Dynamic Light Scattering. Application to Polystyrene Latex Spheres and to Nonionic Microemulsions
}

\author{
A. Eshuis,* G. Harbers, D. J. Doornink, and P. F. Mijnlieff \\ Department of Applied Physics, Twente University of Technology, \\ 7500 AE Enschede, The Netherlands
}

Received October 3, 1984

\begin{abstract}
Information about polydisperse colloidal systems was obtained by dynamic light scattering. The correlation functions obtained were analyzed by the histogram method and the method of cumulants. The former was, as a test, applied to a nearly monodispere polystyrene latex. The agreement between the obtained results and the values given by the supplier was rather good. Therefore, this technique was applied to some nonionic microemulsions to determine their particle size distribution. The results obtained were in agreement with our rheological measurements on these systems published previously. The cumulant method was used to follow the diffusion coefficient of the dispersed particles in microemulsions as a function of temperature.
\end{abstract}

\section{Introduction}

Light scattering has proved to be useful in characterizing colloidal systems. This stems largely from the fact that the wavelength of the radiation used in these experiments is of the same order of magnitude as the dimensions of the dispersed particles. Light scattering has long been used by polymer scientists to determine the average molecular weight of macromolecules. In this technique the intensity of the scattered light is extrapolated to zero scattering angle and zero concentration in a so-called Zimm plot. The same method can be used to obtain dimensions of particles in other systems, as long as they are in the colloidal size range.

With the introduction of the laser a whole new technique became available. Because of the high intensity and optical stability of this light source it is possible to measure fluctuations in the intensity of the scattered light. These can be linked to fluctuations in the dielectric constant of the scattering medium from which, with appropriate models, it is possible to derive the diffusion coefficient of particles in colloidal systems. In the case of spherical particles the Stokes-Einstein relation gives, in sufficiently dilute systems, their radius. This technique is called dynamic light scattering (DLS). ${ }^{1}$

As our research interest lies largely in the field of microemulsions the first technique cannot be applied, since these systems are in general not dilutable. With DLS the diffusion coefficient of particles in a colloidal system can be derived directly from measurements on one single sample. This makes it a very attractive method. However, the diffusion coefficient is determined by the dimensions of the particles and their concentration. Therefore, if one wants to determine these dimensions, interparticle effects must be eliminated.

DLS is most valuable for systems in which the dispersed particles are spheres. It is only for this case that the Stokes-Einstein relation gives reliable information. Although there are a number of systems in which all particles are at least nearly spherical, their radius is usually not uniform. Therefore, the determination of the particle size distribution has recieved much attention. Well-known examples are the cumulant method of Koppel ${ }^{2}$ and the histogram method of Gulari et al. ${ }^{3}$ A review of this field

* Present address: Hoechst Holland NV, Lijndonk 25, $4825 \mathrm{BG}$ Breda, The Netherlands. has been given by Ostrowsky and Sornette. ${ }^{4}$

In this paper these methods will be applied to a diluted nearly monodisperse polystyrene (PS) latex and to nonionic microemulsions. Special emphasis will be placed on the histogram method. The latex has been used because it contains spherical PS spheres of nearly uniform and well-known size, thus providing a test case for the method used. The microemulsions mentioned are much more complicated systems. In the first place they contain three components, one of which is, in fact, a mixture of different related compounds. Second, the dispersed particles are not always spherical. There are, however, circumstances under which it is likely that these systems do contain spheres, making it possible to determine their particle size distribution by the histogram method.

\section{Dynamic Light Scattering of Polydisperse Systems}

Fluctuations in physical quantities are usually represented in terms of correlation functions. The normalized electric field autocorrelation function $g^{(1)}(t)$ of light scattered by a polydisperse colloidal system can be given as $^{2-4}$

$$
g^{(1)}(t)=\int_{0}^{\infty} G(\Gamma) \exp (-\Gamma t) \mathrm{d} \Gamma
$$

in which

$$
\begin{gathered}
\Gamma=q^{2} D \\
q=\frac{4 \pi n}{\lambda} \sin \frac{\theta}{2}
\end{gathered}
$$

and

$$
\int_{0}^{\infty} G(\Gamma) \mathrm{d} \Gamma=1
$$

In these equations $n$ is the refractive index of the continuous phase, $\lambda$ the wavelength of the radiation in vacuum, $\theta$ the scattering angle, $D$ the diffusion coefficient, and $G(\Gamma)$

(1) Berne, B. J.; Pecora, R. "Dynamic Light Scattering"; Wiley: New York, 1976

(2) Koppel, D. E. J. Chem. Phys. 1972, 57, 4814.

(3) Gulari, E.; Gulari, E.; Tsunashima, Y.; Chu, B. J. Chem. Phys. 1979, 70, 3965

(4) Ostrowsky, N.; Sornette, D. In "Photon Correlation Techniques in Fluid Mechanics"; Schulz-DuBois, Ed.; Springer Verlag: New York, 1983. 
the normalized intensity distrubition function, representing the scattering power, of particles of a given size. The magnitude of the electric field of scattered radiation is proportional to the polarizability of the individual particles, which is in its turn proportional to their volume or mass. Because the intensity of electromagnetic radiation is determined by the square of the electric field it follows that the intensity- and the number-distribution function are related, apart from a normalization constant, as

$$
N(a) M^{2}=G(\Gamma)
$$

in which $M$ is the mass of the dispersed particles and $a$ their radius.

If the scattering volume contains a large number of independent scatterers, a condition that usually is satisfied, there is a very simple relation between $g^{(1)}(t)$ and the normalized homodyne intensity autocorrelation function $g^{(2)}(t):^{1}$

$$
g^{(2)}(t)=1+\left[g^{(1)}(t)\right]^{2}
$$

In the case of monodisperse particles it is easy to see that

$$
g^{(2)}(t)=1+e^{-2 \Gamma t}
$$

and when the particles are spheres the Stokes-Einstein relation gives the radius in terms of the Boltzmann constant $(k)$, temperature $(T)$, viscosity of the continuous phase $(\eta)$, and $D$ :

$$
a=\frac{k T}{6 \pi \eta D}
$$

Usually the measured autocorrelation functions contain more than one exponential. If eq 7 and 8 are used to analyse these results, the values for $D$ and $a$ obtained are some kind of a weighted average. ${ }^{5-7}$ Especially for broad distributions they are hard to interpret.

In principle it is possible to determine the complete particle size distribution from dynamic light scattering. Solving $G(\Gamma)$ from eq 1 seems to be the most simple way, meaning that one has to calculate numerically the inverse Laplace transform of $g^{(1)}(t)$. Problems of this type have received much attention and expressions as eq 1 are known as Fredholm integral equations of the first kind. Although their numerical solution is conceptually easy this process is quite unstable. 8 Very high precision in $g^{(1)}(t)$ is needed to get reasonably accurate distributions. ${ }^{10}$

Far more simple is the method of cumulants in which the logarithm of $g^{(1)}(t)$ is written as a sum of terms. ${ }^{2}$

$$
\ln \left[g^{(1)}(t)\right]=\sum_{m=1}^{\infty} K_{m}(\Gamma) \frac{(-t)^{m}}{m !}
$$

in which the factors $K_{m}$ are the $m$ th cumulant and are linked to the moments $\mu_{m}$ of the intensity distribution as

$$
\begin{gathered}
K_{1}=\mu_{1} \quad K_{2}=\mu_{2}-\mu_{1}^{2}=\sigma_{I}^{2} \\
K_{3}=\mu_{3}-2 \mu_{2} \mu_{1}+2 \mu_{3}
\end{gathered}
$$

Thus $K_{1}$ yields the average, $K_{2}$ the standard deviation, and $K_{3}$ some information about the skweness of the distribution mentioned. ${ }^{11}$ Whereas $K_{1}$ can be determined quite

(5) Gulari, E.; Bedwell, B. J. Colloid Interface Sci. 1980, 77, 202. (6) Brown, J. C.; Pusey, P. N.; Goodwin, J. W.; Ottewill, R. H. J. Phys. A.: Math. Gen. 1975, 8, 664

(7) Cebyla, D. J.; Ottewill, R. H.; Ralston, J.; Pusey, P. N. J. Chem. Soc., Faraday Trans. 1 1981, 77, 2585.

(8) McWhirter, J. G., Pike, E. R. J. Phys. A.: Math. Gen. 1978, 11, 1729

(9) McWhirter, J. G. Opt. Acta 1980, 27, 83

(10) Brouwhuis, G. J., private communication, 1982.

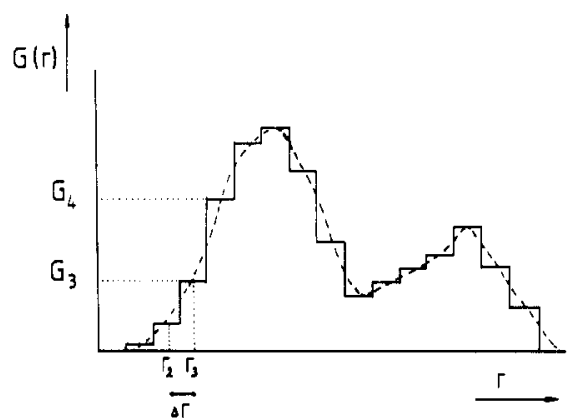

Figure 1. Approximation of an arbitrary function by a histogram.

accurately this is becoming more and more diffucult for the higher cumulants. The error in $K_{2}$ is usually reasonable: about 10-20\% depending on the quality of the masurements. Values for $K_{3}$, obtained by this method, often have very little meaning. This implies that the cumulant method gives reliable information only about the mean and about the standard deviation of a distribution. This is a rather incomplete picture, especially for more complicated distributions.

Sometimes there are reasons to assume that the particle size distribution in a colloidal system is of a certain type representable by a function containing just a few parameters. Values for these can be obtained by fitting measured and calculated correlation functions by least-squares procedures. An example sometimes used in the Schultz distribution with two parameters:

$$
f\left(\frac{a}{\bar{a}}\right)=\frac{1}{z !}\left(\frac{z+1}{\bar{a}}\right)^{z+1} a^{z} \exp \left(-(z+1) \frac{a}{\bar{a}}\right)
$$

in which $\bar{a}$ is the average value for the radius and $z$ is a parameter characterizing the shape. Large values give narrow Gaussian distributions whereas low values of $z$ correspond to broad ones with a positive skewness.

There are many other possibilities, for instance Pearson distribution function. , $^{3,4}$ But all these parameterized distributions share the same disadvantage in that some knowledge about the system is put into the procedure for deriving values of the parameters. Often such information is not available, so that other methods are needed. With the histogram method almost no information is used a priori and it will be described in the next section.

\section{Histogram Method}

The distribution function $G(\Gamma)$ as introduced in eq 1 can be very complex. In principle, however, it is possible to approximate any function by a histogram. An example is shown in Figure 1 where a distribution function is represented by a discrete sum of $m$ terms. This transforms eq 1 into

$$
g^{(1)}(t)=\sum_{j=1}^{m} G_{j} \int_{\Gamma_{j}-(\Delta \Gamma / 2)}^{\Gamma_{j}+(\Delta \Gamma / 2)} \exp (-\Gamma t) \mathrm{d} \Gamma
$$

and consequently

$$
g^{(1)}(t)=\frac{2}{t} \sinh \left(\frac{t \Delta \Gamma}{2}\right) \sum_{j=1}^{m} G_{j} \exp \left(-t \Gamma_{j}\right)
$$

From eq 6 and 13 the homodyne intensity autocorrelation can be obtained, making it possible to derive the unknown coefficients $G_{j}$ from a least-squares fitting procedure. ${ }^{3}$

In fact a histogram is not fundamentally different from other parameterized distribution functions. Its usefulness

(11) Kreyszig, E. "Introductory Mathematical Statistics"; Wiley: New York, 1970 


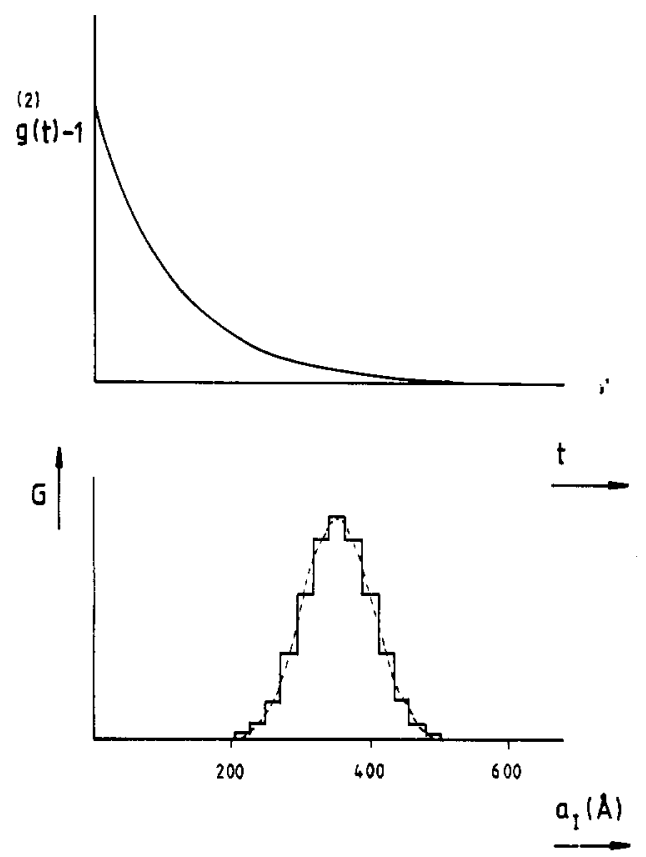

Figure 2. Calculation of a correlation function from a Gaussian distribution function and the distribution obtained from this correlation function by the histogram method.

lies mainly in its universality. But there are three parameters that have to be fixed in the fitting procedure. These are the number of histograms $(m)$, their width $(\Delta \Gamma)$, and the lower limit of the distribution $\left(\Gamma_{1}\right)$. The optimum value for $m$ depends on the quality of the measurements and has to be determined experimentally. For low $m$ the resulting distribution doesn't contain much information and for high $m$ the fitting procedure becomes unstable. Usually the parameters $\Delta \Gamma$ and $\Gamma_{1}$ are chosen in a first attempt such that the actual distribution almost certainly lies in the scanned domain. This gives already some idea of the result and a second run with adjusted values for $\Delta \Gamma$ and $\Gamma_{1}$ gives more details of the desired distribution.

The kind of results obtainable are illustrated by the following highly idealized example, which can serve as a test for the procedure. The broken line in the lower part of Figure 2 represents a Gaussian intensity distribution function with an average radius $\bar{a}_{\mathrm{I}}=350 \AA$ and a standard deviation $\sigma_{\mathrm{I}}=100 \AA$. With eq $8,2,13$, and 6 the corresponding homodyne intensity autocorrelation function $g^{(2)}(t)$ can be evaluated straightforwardly. The net signal part of it, calculated with $T=298 \mathrm{~K}$ and $\eta=0.89 \mathrm{mPa}$ $\mathrm{s}$, is shown in the upper part of Figure 2. Although it looks very smooth it is not completely noiseless, due to rounding errors introduced with the computation. This function forms the input for the procedure to calculate the original distribution back again. The result for $m=13$ is given as the solid line in the lower part of the figure mentioned before.

With $m=15$ this procedure does not converge to a stable solution, due to the inevitable noise. This means that the histogram given contains the maximum amount of information that can be extracted from the correlation function under consideration. Because the noise in measured correlation functions is usually larger than in this example it is to be expected that in those cases the maximum obtainable value of $m$ will be lower.

\section{Polystyrene Latex Spheres}

The previous section provides only a test for the mathematical procedure. In actual measurement, however, at least two complications usually arise. First, there is the
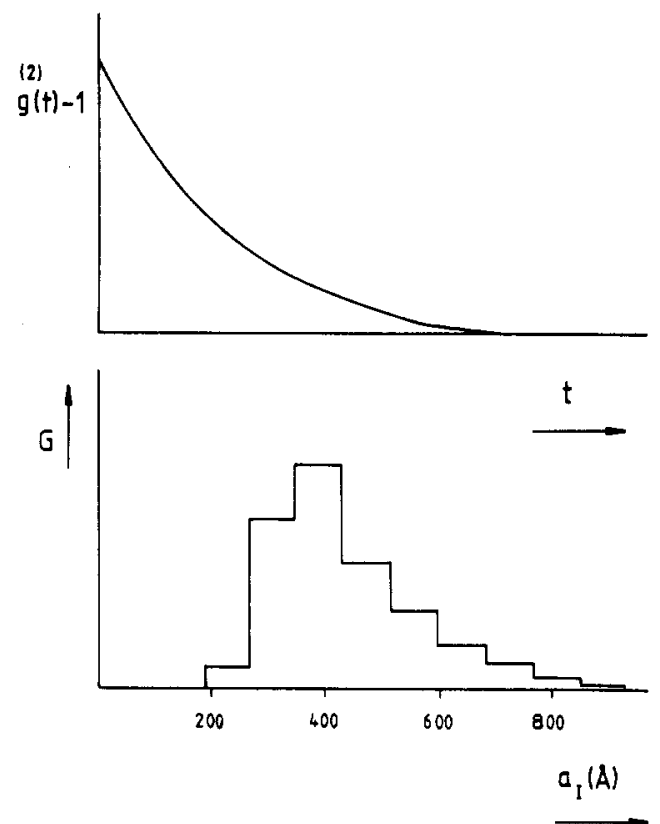

Figure 3. Experimental autocorrelation function and resulting intensity distribution function of a polystyrene latex.

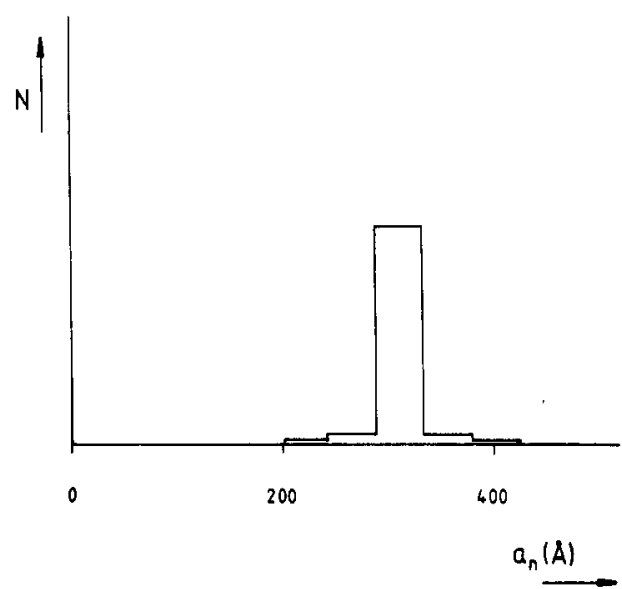

Figure 4. Number distribution function of the polystyrene latex.

inevitable noise on the autocorrelation functions, and, second, the validity of eq 6 is not beyond question. Therefore, to get an impression about the reliability of the results of the histogram method, measurements on a model system are desirable.

Polystyrene latex spheres are ideally suited for this purpose. They were obtained from Polysciences Inc., Warrington, PA, and their radius was given as $330 \AA$ with a standard deviation of about $20 \AA$. The latex as received was diluted a hundred times by triply distilled water so that the volume fraction of the spheres in the final solution was about 0.0002 . The light scattering experiments were performed in the homodyne mode at $25^{\circ} \mathrm{C}$. The working of this equipment was described previously. ${ }^{12}$

The experimentally obtained autocorrelation function of the light scattered by the latex is shown in the upper part of Figure 3. It is the result of about $24 \mathrm{~h}$ of measurements. The normalized intensity distribution function, calculated with $m=9$, is given in the lower part of the same figure. It results in an intensity averaged radius $\bar{a}_{\mathrm{I}}$ $=380 \AA$ and a standard deviation $\sigma_{I}=120 \AA$. By taking into account the scattering power of the individual particles as a function of their radius the number distribution in

(12) Eshuis, A.; Mellema, J. Colloid Polym. Sci. 1984, 262, 159. 
easy to calculate. It is given graphically in Figure 4.

Obviously there are not many particles with a radius larger than $400 \AA$ although Figure 3 shows that the light scattered by these is quite significant. This means that because of the strong dependence of scattering on the radius the resulting histogram representation of the particle size distribution contains fewer details than the intensity distribution function. In spite of this minor drawback it results in a number-averaged mean radius $\bar{a}_{n}$ $=320 \AA$, which corresponds quite well with the value given by the supplier. Our value for the standard deviation, $\sigma_{n}$ $=30 \AA$, is slightly higher than the suppliers one. The overall correspondance, however, may be considered satisfactory.

To get an impression about the reliability of the results obtainable with the cumulant method, the autocorrelation function given in Figure 3 was also used to derive from it the values of $\bar{a}_{\mathrm{I}}$ and $\sigma_{\mathrm{I}}$ with the latter method. We found $\bar{a}_{I}=370 \AA$ and $\sigma_{1}=110 \AA$, and these values agree well with those obtained by the histogram method.

The advantage of the cumulant method is that less accurate correlation functions (obtainable in a shorter time, therefore) are required than for the histogram method. Its disadvantage is that less information is abstracted from the correlation function. Without further information about the distribution the values of $\bar{a}_{\mathrm{I}}$ and $\sigma_{\mathrm{I}}$ do not provide those of $\bar{a}_{\mathrm{n}}$ and $\sigma_{\mathrm{n}}$.

We will use the histrogram method when we need detailed information about a distribution of particles known to be about spherical. The cumulant method will be applied when we are interested in a trend when changing a system parameter.

\section{Nonionic Microemulsions}

General. Microemulsions are colloidal systems composed of water, oil, and one or more surface-active agents. They distinguish themselves from normal emulsions in that they are thermodynamically stable. So the classical picture of an emulsion (spherical droplets dispersed into another liquid and stabilized by a surfactant at the interface) does not necessarily apply to a microemulsion which is defined purely phenomenologically.

In previous papers it was shown that the structure of microemulsion based on the following general type of nonionic surfactants did change considerably as a function of temperature and surfactant to oil ratio. ${ }^{12,13}$

$$
\mathrm{C}_{n} \mathrm{H}_{2 n+1} \mathrm{C}_{6} \mathrm{H}_{4} \mathrm{O}-\left[\mathrm{CH}_{2}-\mathrm{CH}_{2}-\mathrm{O}-\right]_{m} \mathrm{H}
$$

This was established primarily by rheological measurements within their domain of stbility. At relatively high surfactant to oil ratios and low temperatures the results indicated a dispersion of spherical droplets and therefore this part of the phase diagram was called the "sphere region". The temperature of composition region where these systems displayed strong viscoelastic effects was called the "network region".

Two points, however, could not be cleared from the rheological measurements alone. In the sphere region the results were not fully consistent with a dispersion of monodisperse droplets. Spheres with a nonuniform radius could account for this and therefore the determination of the particle size distribution is of the utmost importance. Furthermore, dynamic light scattering may give some information about the structure of these systems at the transition zone between the two subregions.

Microemulsions based on the detergents NNP7 ( $n=9$, $\bar{m}=6.8)$ and NDP8 ${ }^{1} / 2(n=12, \bar{m}=8.3)$ are the subject of investigation in this paper. These surfactants were

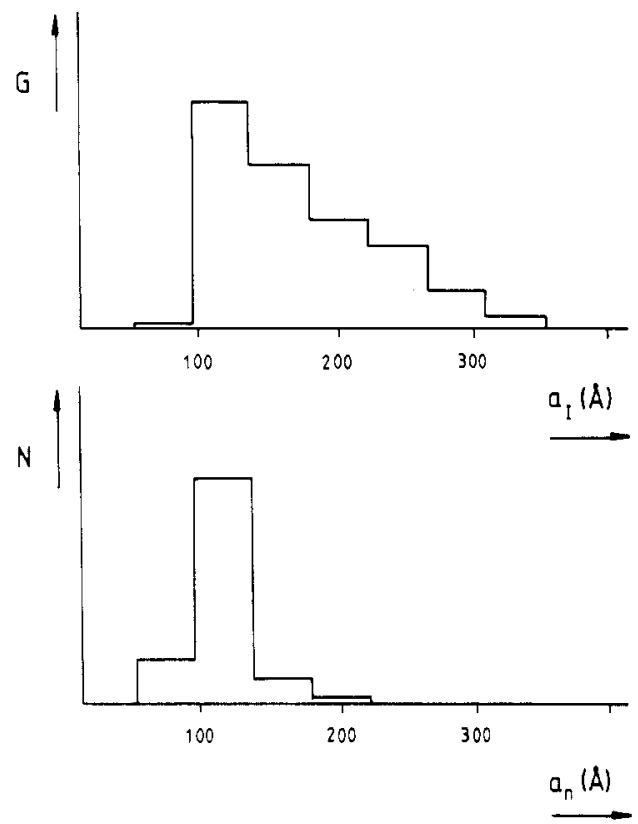

Figure 5. Intensity and number distribution functions of a NNP7 microemulsion. $\phi=0.05$; weight ratio surfactant to $n$-hexane $x$ $=1.3 ; T=19.75^{\circ} \mathrm{C}$.

characterized before. ${ }^{12,13}$ In all cases $n$-hexane acted as the oil phase. To establish the particle size distribution in the sphere region the histogram method was used. With the method of cumulants the transition from spheres to a network was investigated.

Sphere Region. Microemulsions in this part of the phase diagram are easily distinguished from those in the network region in that the viscosity of the systems under these circumstances is much lower. In fact it corresponds to the viscosity of a dispersion of individual droplets. Therefore, our results of dynamic light scattering in this region are analyzed by means of eq 7 . Only in the case of spherical particles the physical meaning of $a$ thus obtained is clear, otherwise equivalent radius results are obtained.

An example of the analysis of a NNP7 system is given in Figure 5 where results are shown for a microemulsion with a composition and at a temperature as indicated in the legend to the figure. The results for a NDP8 $1 / 2 \mathrm{mi}-$ croemulsion are presented in Figure 6.

A small but interesting difference between the two microemulsions is that the NNP7 systems (with $\sigma_{n} \sim 40$ $\AA$ ) are slightly more polydisperse than those based on $\mathrm{NDP}^{1} / 2$ (with $\sigma_{n} \sim 20 \AA$ ). The difference has significance, for the corresponding intensity distributions are very distinct. It is interesting to note that the rheological measurements mentioned before also indicate that NNP7 systems are more polydisperse.

Network Region. Theory of dynamic light scattering in systems that are more complicated than a simple dispersion of spherical particles is not very developed. The Stokes-Einstein relation does not apply and results have to be analyzed in terms of the diffusion coefficient $D$. As long as the system considered is a dispersion of individual particles in a continuous phase the meaning of this parameter is still clear. If, however, some kind of a temporary network exists in solution the interpretation of the diffusion coefficient as measured becomes difficult; just intuitively we tend to relate it to the local mobility of the network.

(13) Eshuis, A., manuscript in preparation

(14) Eshuis, A.; Mijnlieff, P. F., manuscript in preparation. 

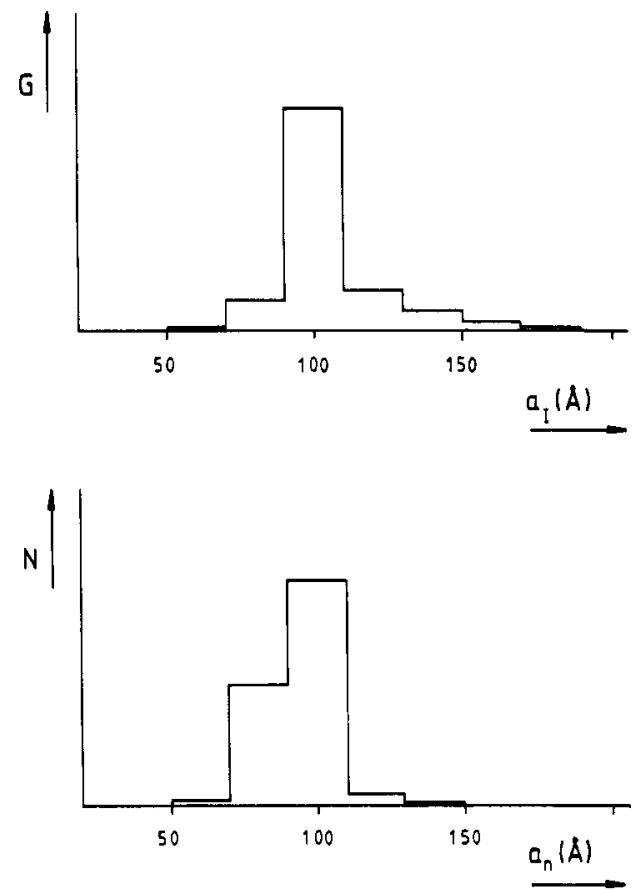

Figure 6. Intensity and number distribution functions of a $\mathrm{NDP}^{1} / 2$ microemulsion. $\phi=0.05 ; x=1.3 ; T=23.09^{\circ} \mathrm{C}$.

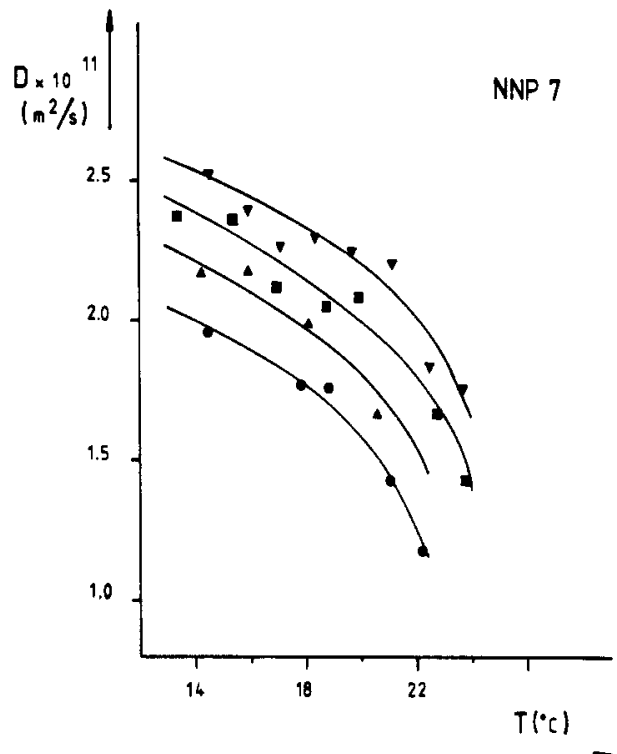

Figure 7. Diffusion coefficient as a function of temperature and volume fraction for NNP7 microemulsions, $x=1.4$. (ब) $\phi=0.060$; (A) $\phi=0.100 ;(\square) \phi=0.139 ;(\nabla) \phi=0.178$.

Data on the diffusion coefficient, as obtained by the cumulant method, in NNP7 and NDP8 $1 / 2$ systems in their domain of stability are presented in Figures 7 and 8, respectively, in which $x$ is the weight ratio of surfactant to oil. The patterns recognizable in these two sets of graphs are rather different. Although the points show a large amount of scatter it is clear that in Figure $7 D$ decreases monotonously with temperature whereas a maximum is observed in Figure 8.

In a previous paper it was shown that the decrease in $D$ can be attributed to the formation of nonspherical particles. ${ }^{13}$ This transition seems to be induced by con-

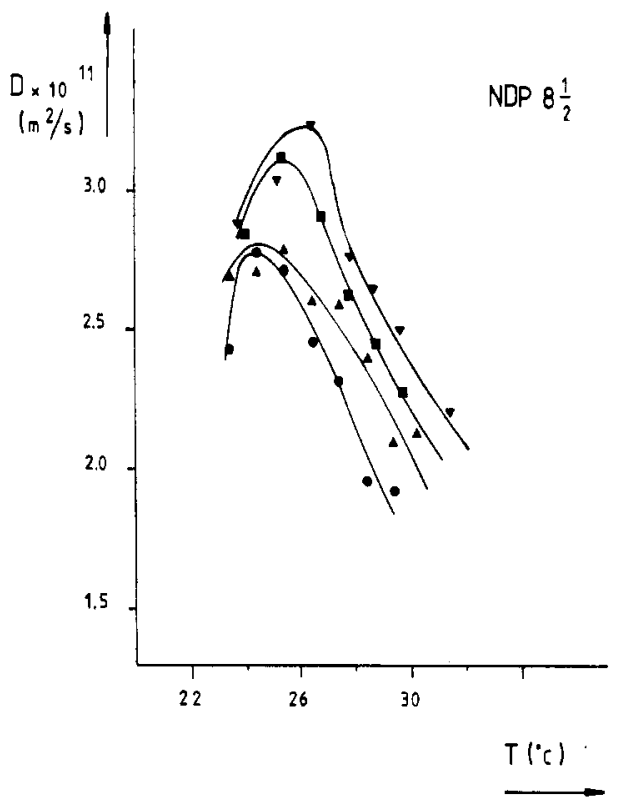

Figure 8. Diffusion coefficient as a function of temperature and volume fraction for NDP8 $1 / 2$ microemulsions, $x=1.3$. (๑) $\phi=$ $0.063 ;(\Lambda) \phi=0.104 ;(\square) \phi=0.144 ;(\nabla) \phi=0.184$.

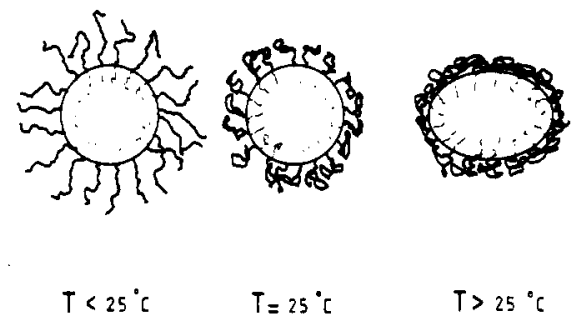

Figure 9. Influence of temperature on the conformations of the polyethylene oxide chains and on the shape of the dispersed particles.

formational changes in the ethylene oxide part of the surfactant molecules, as demonstrated for aqueous polyethylene oxide solutions in ref. 14. The results presented in Figure 8 can be explained in the following way. At the lowest temperature the solubility of the PEO chains is highest, making the hydrodynamic radius of the particle larger than the radius of the $n$-hexane core (Figure 9). When temperature is increased self-association of the chains gives rise to a lower value for this hydrodynamic radius and thus a higher diffusion coefficient. At still higher temperatures the surface of the particles becomes so crowded that it tends to increase resulting in otherwise shaped entities whose diffusion coefficients are lower than those of spheres, like in the case of NNP7 systems.

The observed initial increase of $D$ with rising temperature can also be explained with a redispersion of the spheres to smaller ones. Our results on the dynamic mechanical behavior of NDP8 $1 / 2$ systems do, however, indicate otherwise. The large difference in observed behavior between NNP7 and NDP8 $1 / 2$ is not too surprising because it is shown tht small changes in the number of ethylene oxide units in the hydrophylic chain can have a remarkable influence as temperature dependence is concerned. Preliminary results on NNP12 systems show that still larger changes are observed.

Registry No. Polystyrene, 9003-53-6. 